01

\title{
O перераспределении примеси в коллоидных смесях
}

\author{
(C) И.Н. Черепанов
}

e-mail: info@psu.ru

(Поступило в Редакцию 5 декабря 2017 г.)

Рассмотрены процессы перераспределения примеси наночастиц в коллоидной смеси при двух постановках задачи: отстаивание смеси в узком канале в горизонтальном положении, а затем приведенным в вертикальное, а также при переливании смеси из емкости в узкий канал. Течение, приводящее систему к устойчивому состоянию со строго вертикальным градиентом плотности, искажает начальное барометрическое распределение примеси наночастиц. Методами численного моделирования показано, что в обоих случаях начальная неоднородность смеси в значительной мере сохраняется.

DOI: 10.21883/JTF.2018.12.46775.2589

\section{Введение}

Смеси компонентов с различной плотностью, находящиеся в поле тяжести, подвержены гравитационной седиментации, когда при длительном отстаивании концентрация тяжелой компоненты увеличивается в нижних слоях смеси [1]. В случае молекулярных смесей, в которых смешиваемые компоненты диспергированны на молекулярном уровне, эффект гравитационного расслоения проявляется довольно слабо, и может быть заметен только при очень больших вертикальных размерах емкости.

С другой стороны, в грубодисперсных смесях, когда частицы имеют большой размер (более $1000 \mathrm{~nm}$ ), энергия теплового движения молекул среды носителя не способна поддерживать примесные частицы во взвешенном состоянии, и с течением времени они выпадают на дно в виде осадка.

Особое место в смесях занимают коллоидные растворы, другое название наножидкости, или золи [1]. К таким смесям относятся системы, в которых энергия теплового движения молекул сопоставима с энергией на- ночастиц в гравитационном поле Земли [2]. В этом случае примесь может вечно находиться во возвещенном состоянии, не выпадая в осадок. Однако распределение концентрации наночастиц по вертикали является существенно неоднородным в высоких сосудах. Величина неоднородности распределения примеси характеризуется седиментационной длиной - высотой, на которой концентрация уменьшается в $e$ раз [2]. Данный параметр определяется соотношением тепловой энергии молекул и энергией гравитационного поля:

$$
l_{\text {sed }}=\frac{k_{\mathrm{B}} T}{\rho_{s}-\rho_{f} g V_{s}},
$$

где $V_{s}$ - характерный объем одной частицы, $\rho_{s}$ - плотность частиц, $\rho_{f}-$ плотность жидкости носителя, $k_{\mathrm{B}}-$ постоянная Больцмана, $T$ - абсолютная температура, $g$ - ускорение свободного падения. Для реальных жидкостей данный параметр может иметь порядок значений от единиц [3] до нескольких десятков сантиметров [4].
При рассмотрении задач тепловой конвекции малая неоднородность поля концентрации может оказаться существенной. Коэффициент концентрационного расширения коллоидных систем превосходит коэффициент теплового расширения на несколько порядков. Таким образом, неоднородность концентрации в сотые доли процента создает возмущения плотности, сопоставимые с тепловым расширением.

В экспериментальных наблюдениях конвекции наножидкостей существуют течения, которые не проявляются в однородных средах: бегущие волны [3,5], перебросовые течения [6,7], локализованные структуры [8]. Теоретические исследования показывают, что большинство подобных течений обусловлены концентрационной неоднородностью смеси, которые вызываются гравитационным $[9,10]$ или термодиффузионным [11] разделением компонент, магнетофорезом [12], а также их совместным влиянием [13].

Процесс седиментации наночастиц в поле тяжести протекает довольно медленно, ввиду малого значения коэффициента диффузии. Теоретическое рассмотрение процесса гравитационного разделения проведено в работе [14], а также изучалось экспериментально в работе [4].

Разделение смеси происходит существенно не равномерно как во времени, так и в пространстве. Неоднородность распределения примеси сначала проявляется вблизи вертикальных границ сосуда, а затем распространяется в толщу жидкости. При этом в сосудах с большим вертикальным размером вдали от горизонтальных границ наблюдается однородное распределение концентрации в течении длительного времени $[4,14]$.

Если к системе с таким профилем концентрации приложить продольную разность температуры, то нарушается условие отсутствия конвекции [15]. Вблизи границ сосуда, где жидкость неоднородна по концентрации, возникшее течение за малое время сформирует такое поле концентрации, при котором горизонтальный градиент плотности будет близок к нулю, т.е. изменение плотности за счет теплового расширения будет компенсироваться концентрационной неоднородностью. В центре слоя, где смесь однородна по концентрации, 


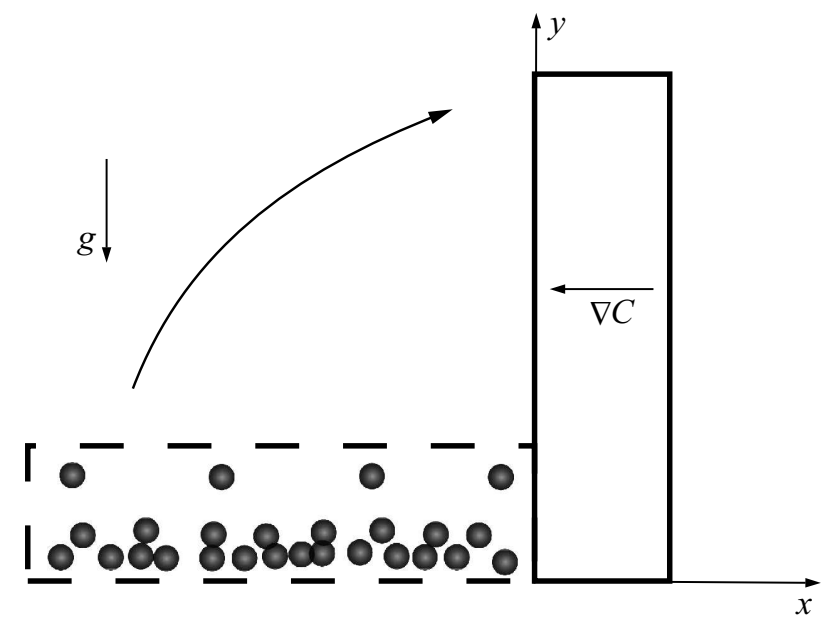

Рис. 1. Геометрия задачи 1.

механизмы компенсации теплового расширения отсутствуют, и возникает интенсивное течение.

В работе [16] показано, что при боковом обогреве стратифицированной смеси может существовать устойчивое состояние с очень слабым течением, не влияющим на процессы теплопереноса. При этом конвективный перенос примеси имеет значение порядка диффузионного потока.

Для ускорения формирования экспоненциального профиля в узком канале жидкость может отстаиваться в горизонтальном положении, а затем емкость приводится в вертикальное положение [17] (рис. 1). При этом остается неясным, насколько сохраняется профиль концентрации, и как параметры жидкости влияют на процесс переливания.

В другом случае коллоид может длительное время находиться в емкости с малым вертикальным размером, а затем переливаться в экспериментальную ячейку.

В настоящей работе рассматриваются результаты численного моделирования процессов перераспределения кон- центрации при повороте плоского горизонтального канала со стратифицированной коллоидной смесью, а также процессы перетекания коллоида в тонкий канал из емкости меньшей высоты.

\section{Постановка задачи}

При рассмотрении коллоидных смесей будем полагать, что наночастицы примеси движутся только со средой, и облако частиц не может двигаться относительно жидкости носителя (диффузионное приближение) [18].

Плотность смеси жидкости и твердых частиц описывается выражением:

$$
\rho=\varphi \rho_{s}+(1-\varphi) \rho f,
$$

где $\rho$ - плотность смеси, $\varphi-$ объемная концентрация, $\rho_{f}, \rho_{s}-$ плотности жидкой и твердой фаз соответственно. В терминах массовой концентрации плотность запишется следующим выражением

$$
\rho=\left(\frac{C}{\rho_{s}}+\frac{1-C}{\rho_{f}}\right)^{-1} .
$$

Произведем разложение данного выражения в окрестности некоторого среднего значения концентрации $\bar{C}$ с точность до второго порядка:

$$
\rho=\rho_{0}+\left(\frac{\partial \rho}{\partial C}\right)_{C=\bar{C}}(C-\bar{C})+\frac{1}{2}\left(\frac{\partial^{2} \rho}{\partial C^{2}}\right)_{C=\bar{C}}(C-\bar{C})^{2} .
$$

Зависимость (1) можно считать линейной при выполнении условия

$$
\left|\frac{\partial \rho}{\partial C}(C-\bar{C})\right| \gg\left|\frac{1}{2} \frac{\partial^{2} \rho}{\partial C^{2}}(C-\bar{C})^{2}\right|,
$$

которое можно привести к виду

$$
\left|\frac{\rho_{0}}{\rho_{s}}\left(1-\frac{\rho_{s}}{\rho_{f}}\right)(C-\bar{C})\right| \ll 1,
$$

где $\rho_{0}=\rho(C=\bar{C})$ - плотность коллоида при среднем значении концентрации. Если рассматривать коллоидную смесь с $\rho_{s} \approx 2 \mathrm{~g} / \mathrm{cm}^{3}$ и $\rho_{f} \approx 1 \mathrm{~g} / \mathrm{cm}^{3}$, рассматриваемую в работе [19], то условие примет вид

$$
(C-\bar{C}) \ll 1.5
$$

Следовательно, линейная аппроксимация плотности по концентрации остается справедливой, если отклонения концентрации от среднего значения на порядок меньше 1.5 в терминах массовой доли, т.е. не превосходят 15\%. Подобная оценка для магнитной жидкости, используемой в $[20,21]$, для которой $\rho_{s} \approx 5 \mathrm{~g} / \mathrm{cm}^{3}$, $\rho_{f} \approx 0.7 \mathrm{~g} / \mathrm{cm}^{3}$ и $\rho_{0} \approx 1.4 \mathrm{~g} / \mathrm{cm}^{3}$, дает ограничение в $6 \%$

При рассмотрении течений смеси будем полагать, что отклонение плотности обусловлено только концентрационной неоднородностью. Уравнение состояния коллоидной смеси запишем в виде

$$
\rho=\rho_{0}(1+\beta(C-\bar{C})),
$$

где $\rho_{0}-$ среднее значение плотности, $\beta=\frac{1}{\rho_{0}} \frac{\partial \rho}{\partial C}-$ коэффициент концентрационного расширения, $C$ - $\frac{\partial C}{\rho_{0}}$ зачение массовой концентрации наночастиц. Система уравнений, описывающая концентрационную конвекцию коллоидной смеси $[9,15]$, запишется в виде

$$
\begin{gathered}
\frac{\partial \mathbf{v}}{\partial t}+(\mathbf{v} \nabla) \mathbf{v}=\frac{1}{\rho_{0}} \nabla p+v \Delta \mathbf{v}+\mathbf{n}_{g} g \rho_{0}(1-\beta(C-\bar{C})), \\
\nabla \mathbf{v}=0, \\
\frac{\partial C}{\partial t}(\mathbf{v} \nabla) C=D\left(\Delta C+\frac{1}{l_{\text {sed }}} \mathbf{n}_{g} \nabla C\right),
\end{gathered}
$$

где введены обозначения: $v$ - кинематическая вязкость, $D$ - коэффициент диффузии, $l_{s e d}-$ длина седиментации, $\mathbf{n}_{g}=(0-1.0)-$ единичный вектор вдоль направления силы тяжести. 
При отсутствии макроскопического течения $(\mathbf{v}=0)$ распределение концентрации в равновесии описывается выражением

$$
C=\frac{\bar{C} h}{l_{\text {sed }}} \frac{\exp \left(-y / l_{\text {sed }}\right)}{1-\exp \left(-h / l_{\text {sed }}\right)} .
$$

При данном распределении максимальное отклонение концентрации от среднего значения будет достигнуто на нижней границе сосуда. Тогда условие (2) выполняется при

$$
\bar{C}-\frac{\bar{C} h}{l_{\text {sed }}} \frac{1}{\left(1-\exp \left(-h / l_{\text {sed }}\right)\right)} \ll 1 .
$$

Если определить требование малости как различия на порядок (в десять раз), тогда область, в которой сделанные предположения являются справедливыми, можно ограничить условием

$$
\bar{C}<0.1\left(1-\frac{h / l_{\text {sed }}}{1-\exp \left(-h / l_{\text {sed }}\right)}\right)^{-1} .
$$

Данная область изображена на рис. 2. В дальнейшем рассмотрении мы ограничимся максимальным отношением $h / l_{\text {sed }}=0.4$. При таких параметрах максимальная концентрация коллоида не должна превышать $50 \%$ массовой доли.

Вязкость дисперсных систем зависит от концентрации примеси. В случае грубодисперсных взвесей вязкость хорошо описывается формулой Эйнштейна [22]

$$
\eta=\eta_{0}(1+A \phi),
$$

где $A-$ коэффициент, зависящий от формы взвешенных в жидкости частиц $(A=2.5$ для сферических частиц). Для коллоидных систем вязкость не подчиняется закону (6). Экспериментальные исследования показывают, что вязкость коллоидной смеси можно описывать квадратичным выражением [23]

$$
\eta=\eta_{0}\left(1+a \phi+b \phi^{2}\right),
$$

где коэффициенты $a$ и $b$ - зависят от множество факторов: размера, формы и материала частиц, также от

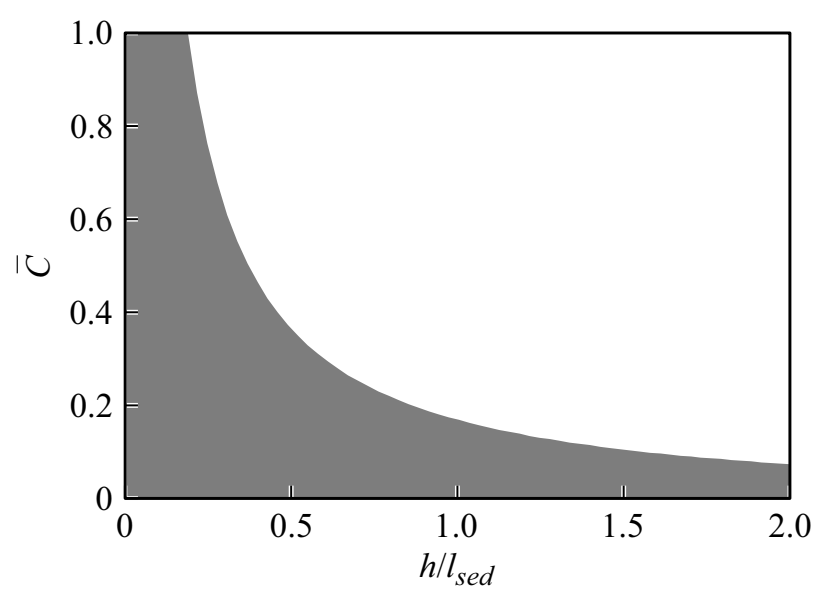

Рис. 2. Область применимости приближения Буссинеска.

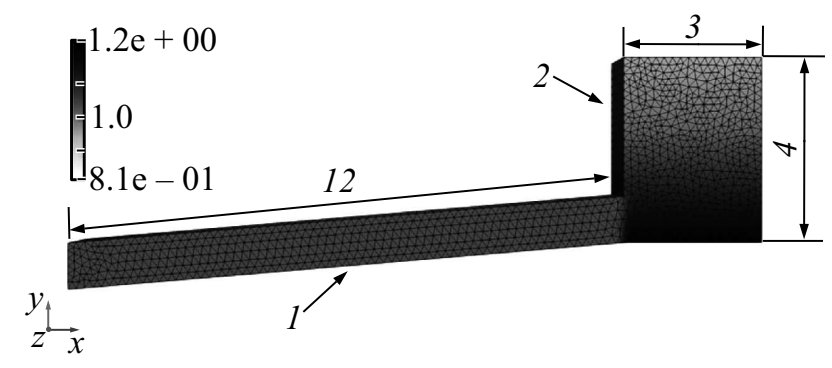

Рис. 3. Расчетная область. Цветом отображено начальное распределение концентрации (описание в тексте).

жидкости носителя и нескольких прочих факторов [23]. В экспериментальных исследования данные коэффициенты имеют значения $a \sim 10, b \sim 10^{2}[19,24,25]$. Рacсмотрим отклонения вязкости, обусловленные малыми отклонениями концетрации от среднего значения:

$$
\eta=\eta_{0}\left(1+a(\bar{\phi}+\delta \phi)+b(\bar{\phi}+\delta \phi)^{2}\right) .
$$

Пренебрегая квадратичным по концентрационному отклонению слагаемым, получим

$$
\eta=\eta_{0}\left(1+a \bar{\phi}+b \bar{\phi}^{2}+(a+2 b \phi) \delta \phi\right) .
$$

Следовательно, зависимость вязкости от концентрации является не существенной при

$$
\frac{\delta \phi}{\bar{\phi}} \ll \frac{1 / \bar{\phi}+a+b \bar{\phi}}{(a+2 b \bar{\phi})} .
$$

Если рассматривать среднюю объемную концентрацию смеси порядка $\phi \approx 10 \%$, то условие (7) для жидкости [24] примет вид $\delta \phi / \bar{\phi} \ll 0.7$, а для [19] $\delta \phi / \bar{\phi} \ll 1$. Учитывая, что относительные отклонения объемной и массовой концентрации совпадают

$$
\frac{\delta \phi}{\bar{\phi}}=\frac{\delta C}{\bar{C}},
$$

условие (7) будет заведомо выполняться при выполнении условий (2), (5), следовательно, в рассматриваемом случае вязкость можно полагать постоянной, не зависящей от концентрации.

Введем безразмерные переменные, выбрав следующие масштабы: длины - ширину канала $h$, времени $h^{2} / v$, давления - $\rho_{0} v^{2} / h^{2}$, концентрации - среднюю концентрацию $\bar{C}$. В безразмерных переменных система уравнений (3), (4) примет вид

$$
\begin{gathered}
\frac{\partial \mathbf{v}}{\partial t}+(\mathbf{v} \nabla) \mathbf{v}=\nabla p+\Delta v+\mathbf{n}_{g}\left(G_{d}(C-\bar{C})\right), \\
\nabla \mathbf{v}=0, \\
\frac{\partial C}{\partial t}+(\mathbf{v} \nabla C)=\frac{1}{S c}\left(\Delta C+\frac{1}{l} \frac{\partial C}{\partial y}\right),
\end{gathered}
$$

где введены следующие безразмерные параметры: концентрационное число Грасгофа $G_{d}=\frac{g \beta h^{4} \bar{C}}{v^{2} l_{\text {led }}}$, Шмидта $S c=v / d[15]$, безразмерная длина седиментации $l=l_{\text {sed }} / h$. 


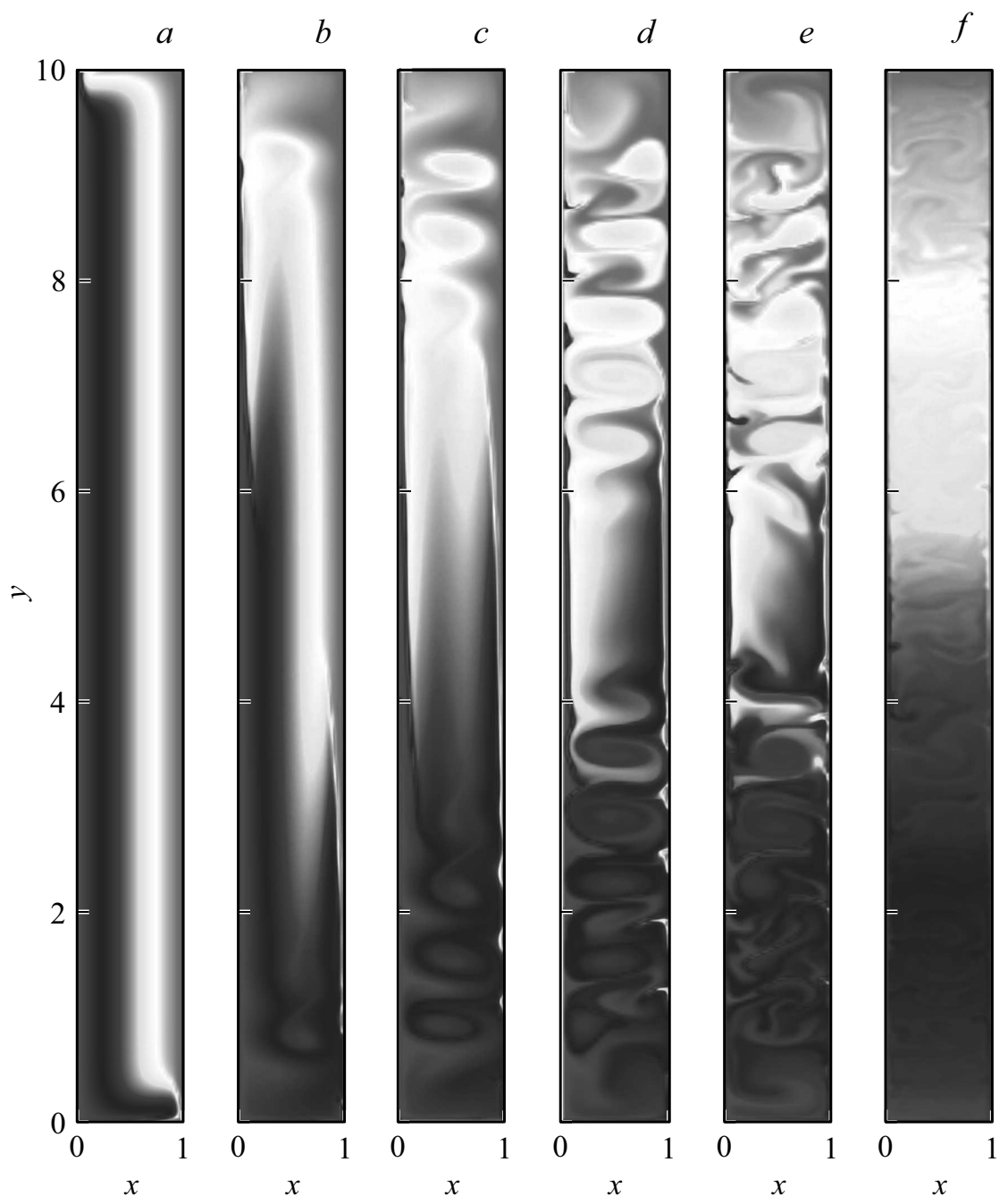

Pис. 4. Поля распределения концентрации для моментов времени, $t: a-1 \cdot 10^{-2}, b-4 \cdot 10^{-2}, c-6 \cdot 10^{-2}, d-8 \cdot 10^{-2}, e-$ $10 \cdot 10^{-2}, f-27 \cdot 10^{-2}$ при $G_{d}=8 \cdot 10^{4}$.
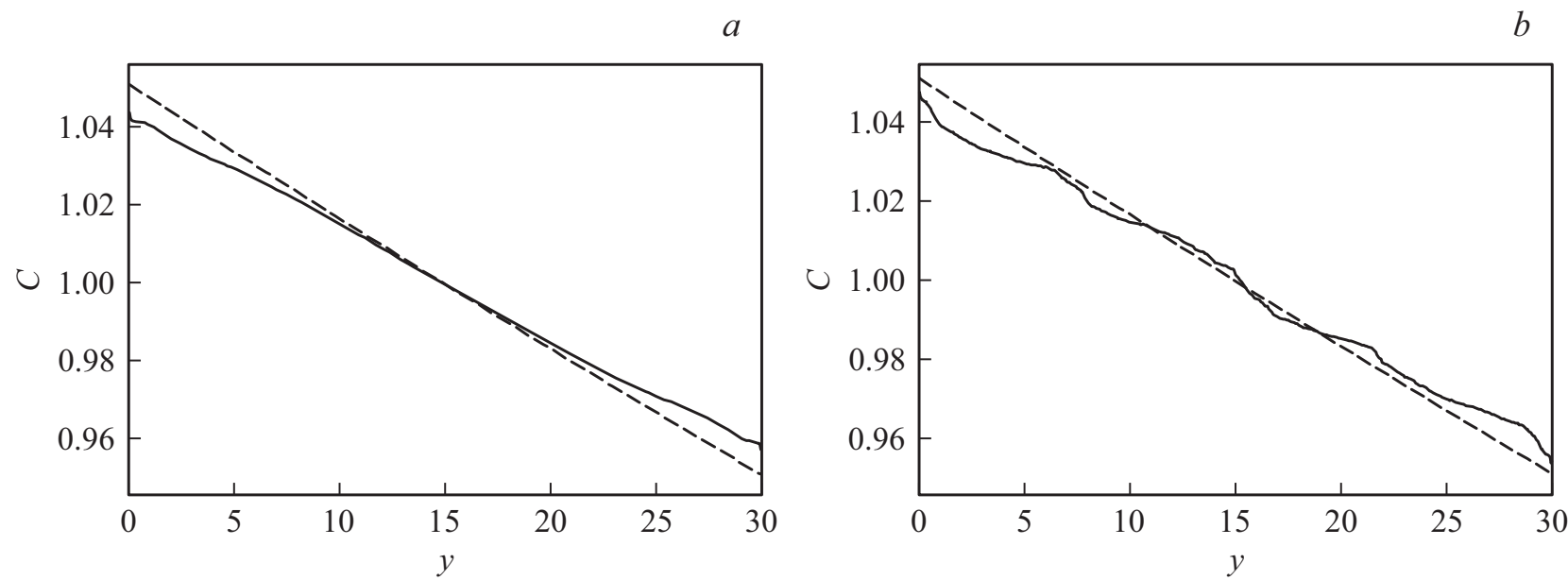

Рис. 5. Конечный профиль концентрации в центре ячейки при: $a-$ ламинарном $G_{d}=5 \cdot 10^{3}$ и $b-$ турбулентном течении $G_{d}=80 \cdot 10^{3}$. Штриховой линией обозначено точное распределение (8), которое могло быть достигнуто в случае абсолютного отсутствия перемешивания. 
На границах полости ставятся условия прилипания для скорости и обращение в нуль нормальной составляющей потока вещества

$$
\begin{gathered}
\mathbf{v}=0, \\
\left(\nabla C+\frac{1}{l} C \mathbf{n}_{g}\right) \mathbf{n}_{s}=0,
\end{gathered}
$$

где $\mathbf{n}_{s}$ - единичный вектор нормали к поверхности.

Вычислительный алгоритм был реализован на открытой платформе решения задач динамики сплошных сред OpenFoam.

Расчетная область для геометрии плоского канала строилась при помощи утилиты blockMesh, входящей в состав пакета OpenFoam, шаг расчетной сетки при этом составлял $1 / 30$.

Для построения более сложной геометрии перетекания жидкости из сосуда в плоский канал применялась программа Gmsh. Граничные условия для концентрации задавались при помощи пакета расширений swek4foam c использование типа граничных условий grooverBC.

Моделирование производилось для двух случаев. В первом случае рассматривалась прямоугольная ячейка с соотношением сторон 1 к 10, в которой распределение концентрации вдоль оси $x$ задалось выражением

$$
C=\frac{1}{l_{\text {sed }}} \frac{\exp \left(-x / l_{\text {sed }}\right)}{1-\exp \left(-1 / l_{\text {sed }}\right)} .
$$

Данная постановка соответствует ситуации, в которой сосуд длительное время находился в горизонтальном положении, а затем был повернут на 90 [17].

Во втором случае рассматривалась более сложная геометрия (рис. 3), в которой плоский канал с соотношением сторон 1 к 12 заполнен однородной по концентрации золью. В сосуде 2 с соотношением сторон 4 к 3 находится стратифицированная суспензия. Данный случай соответствует заполнению экспериментальной ячейки из емкости, в которой образец отстаивался длительное время.

\section{Результат}

\section{Поворот плоского канала}

Рассмотрим случай, когда тонкий канал, заполненный коллоидной смесью, долгое время отстаивался в горизонтальном положении, а затем был приведен в вертикальное состояние (рис. 1). В начальный момент времени, после приведения канала в вертикальное состояние, будет наблюдаться распределение концентрации вида

$$
C(x, y)=\frac{1}{l} \frac{\exp (-x / l)}{1-\exp (-1 / l)} .
$$

Подобное состояние является конвективно неустойчивым, и начинается макроскопическое движение жидкости. В области малых значений параметра $G_{d}$ течение

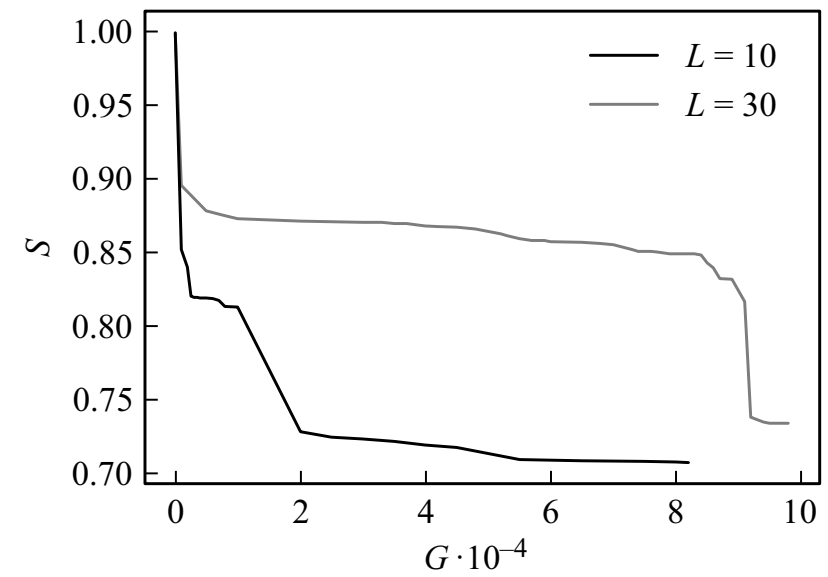

Рис. 6. Значения усредненного вертикального градианта плотности в зависимости от числа $G_{d}$ для двух значений высоты канала $L_{y}=10$ и 30 .

остается ламинарным. Если же $G_{d}$ больше некоторого критического значения, то процесс перераспределения примеси происходит с образованием коротковолновой конвекции.

Пример эволюции поля концентрации при турбулентном течении, с образованием группы вихрей вдоль сосуда приведен на рис. 4. Конвективные валы начинают формироваться вблизи горизонтальных границ полости, практически одновременно в верхней и нижней частях. Затем возмущения распространяются от краев к центру слоя. Возникшие вихри имеют одинаковую закрутку и частично перемешивают наножидкость, что приводит к ослаблению градиента концентрации. Процесс перераспределения примеси протекает довольно быстро и на временах порядка нескольких десятков единиц гидродинамического времени течение полностью затухает.

На рис. 5 приведено распределение примеси по высоте, по истечению времени $t=10$ для турбулентного $G_{d}=80 \cdot 10^{3}$ и ламинарного перетекания $G_{d}=5 \cdot 10^{3}$. Пунктирной линией обозначено точное экспоненциальное распределение, которое могло быть достигнуто в случае абсолютного отсутствия перемешивания

$$
C_{0}(x, y)=\frac{1}{l} \frac{\exp \left(-y /\left(L_{y} l\right)\right)}{(1-\exp (-1 / l))} .
$$

Отметим, что это не конечное состояние системы. Диффузионный массообмен на временах порядка $t=10^{4}$ приведет систему к равновесному распределению, которое описывается выражением

$$
C_{0}(x, y)=\frac{L_{y}}{l} \frac{\exp (-y / l)}{\left(1-\exp \left(-L_{y} / l\right)\right)} .
$$

В турбулентном случае профиль имеет значительные искажения. Градиент плотности при этом всюду направлен вниз, и такое состояние является конвективно устойчивым. Малые флюктуации распределения примеси существуют продолжительное время ввиду малого коэффициента диффузии. 
Для анализа интенсивности перемешивания коллоида воспользуемся усредненным вертикальным градиентом концентрации, нормированным на равновесный градиент, усреднение производилось по обеим координатам:

$$
S=\frac{1}{L_{x} L_{y}} \frac{\iint \frac{\partial C}{\partial y} d x d y}{\iint \frac{\partial C_{0}}{\partial y} d x d y},
$$

где $C_{0}$ - описывается формулой (8). Данный параметр показывает на сколько уменьшится вертикальный градиент концентрации по сравнению с начальным распределением.

Значения $S$ при разных числах Грасгофа приведены на рис. 6. Расчеты были произведены для двух полостей длиной $L_{y}=10$ и 30 . Видно, что при малых $G_{d}$ градиент значительно уменьшается, а затем наблюдается небольшое плато. При $G_{d}<4 \cdot 10^{4}$ течение имеет ламинарных характер. Отметим, что даже при ламинарном течении средний градиент концентрации уменьшается значительно, а также имеет отклонения от экспоненциального распределения вблизи горизонтальных границ емкости (рис. 5).

\section{Заполнение канала стратифицированной наножидкостью}

Рассмотрим случай перетекания стратифицированной наножидкости из сосуда в плоский канал, расположенный под малым наклоном к горизонтали. Геометрия задачи и расчетная сетка приведены на рис. 3. Плоский канал 1 в начальный момент времени заполнен однородной по концентрации суспензией. В емкости 2 находится стратифицированная суспензия с распределение концентрации по высоте (9), в котором $L_{y}=4$. В начальный момент времени сосуды разделены непроницаемой перегородкой. После удаления перегородки на границе сосудов 1 и 2 возникает продольный градиент концентрации, поэтому данное состояние является неустойчивым и возникает макроскопическое движение жидкости. Более

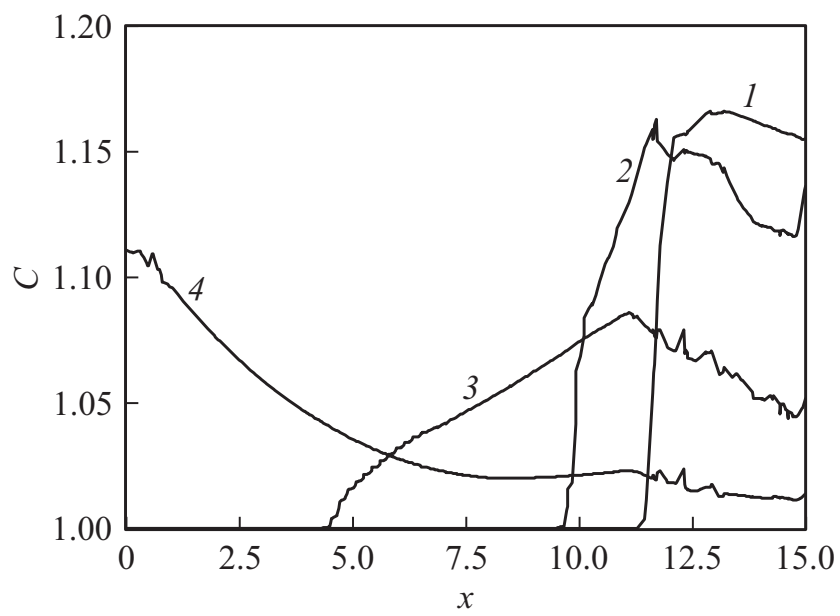

Рис. 7. Профиль концентрации при $G_{D}=5 \cdot 10^{3}$ для моментов времени, $t: 1-0,2-0.12,3-0.5,4-2.5$.

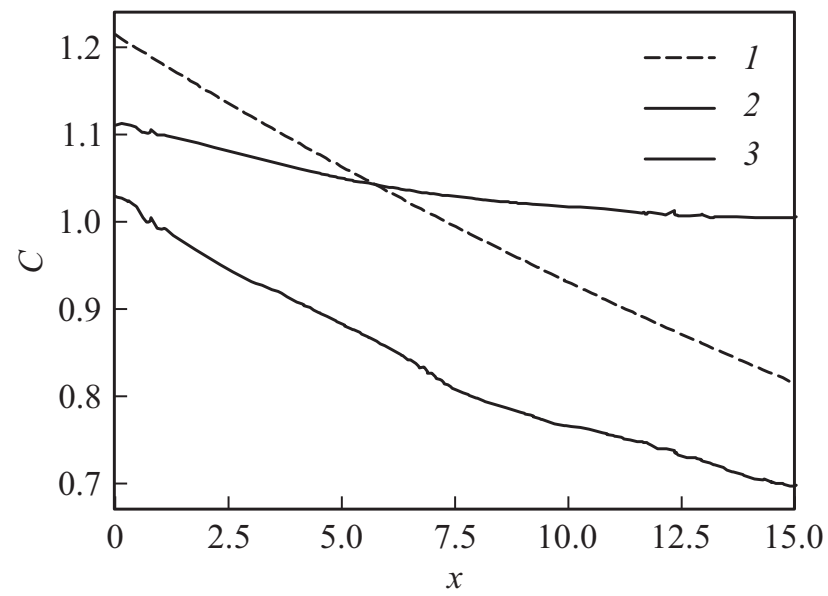

Рис. 8. Профиль концентрации вдоль длинной оси канала. Кривая 1 соответствует точному распределению концентрации 1, если бы жидкость перетекала без перемешивания. Кривые 2 и 3 отображают профиль концентрации для $G_{D}=10^{4}$ при значении концентрации в сосуде в 1 и 0.7 соответственно.

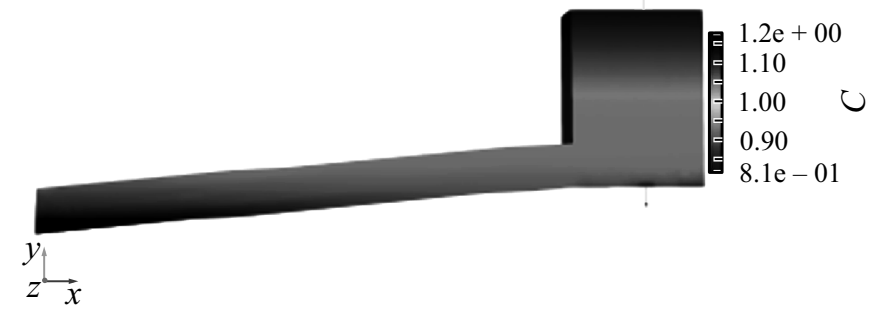

Рис. 9. Поле концентрации после затухания течения.

концентрированная коллоидная смеси стекает по нижней стенке узкого канала. Скорость течения зависит от числа $G_{d}$. Так, при $G_{d}=2 \cdot 10^{3}$ процесс конвективного переноса примеси занимает порядка 5 единиц времени, а при $G_{d}=80 \cdot 10^{3}$ менее единицы.

Эволюция профиля концентрации вдоль плоского канала приведена на рис. 7. Плоский канал 1 имеет длину в 12 единиц, поэтому на рис. 7 область от 12 до 15 находится внутри сосуда 2. Видно, что более тяжелая концентрированная смесь начинает перетекать в плоский канал. При этом за время $t=2.5$ вдоль канала устанавливается профиль концентрации, близкий к экспоненциальному.

Минимальное значение концентрации в канале 1 выше единицы. Очевидно, это связано с тем, что средняя концентрация в начальный момент времени была равной единице, и смесь с концентрацией, меньше единицы, осталась в верхней части емкости 2, так как является более легкой.

Распределение примеси в конечном состоянии для двух значений начальной средней концентрации в канале 1 (распределение концентрации в канале 2 является одинаковым для обоих случаев) и числе $G_{d}=10^{4}$ приведено на рис. 8. Кривая 1 соответствует экспоненци- 


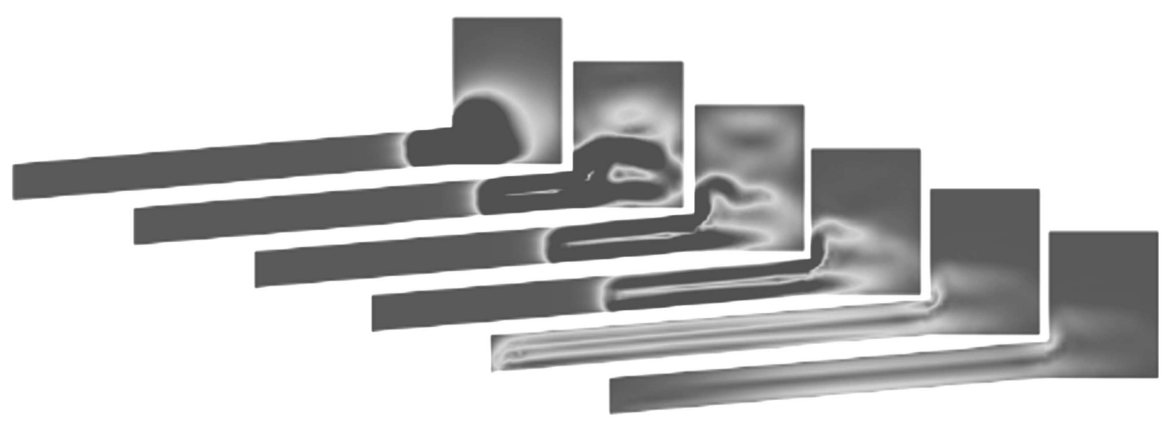

Рис. 10. Амплитуда скорости течения в различные моменты времени.

альному распределению (9) и показывает профиль, если бы перенос жидкости между емкостями произошел без перемешивания и прочих искажений. Кривая 2 отображает профиль концентрации в случае, если безразмерная концентрация в канале 1: $C=1$, т.е. равна средней концентрации в сосуде 2. Видно, что профиль близок к экспоненциальному, однако наименьшее значение концентрации выше единицы, а следовательно, эффективный седиментационный градиент значительно уменьшается. Кривая 3 соответствует начальному значению концентрации в канале 1 , равной $C=0.7$ (напомним, что безразмерная концентрация нормирована на среднее значение $\bar{C}$ ). В этом случае средняя концентрация примеси в канале понижается, и кривая сдвигается вниз. Данный факт обусловлен конвективным перемешиванием жидкости, находящейся в емкости и канале. Однако при такой концентрации перепад концентраций в нижней и верхней частях канала значительно выше.

Карта распределения концентрации после затухания течения приведена на рис. 9.

Сильное перемешивание смеси объясняется наличием очень интенсивного течения на границе каналов. Амплитуда скорости для различных моментов времени приведена на рис. 10.

\section{Заключение}

Проведенное численное моделирование концентрационных течений показывает, что стратифицированное распределение примеси в значительной мере сохраняется как при повороте емкости, так и при переливании смеси между емкостями.

В случае поворота плоской ячейки со стратифицированного коллоида в области малых чисел диффузионного числа Грасгофа наблюдается ламинарное движение жидкости. При больших числа Грасгофа течение является турбулентным. По мере увеличения числа Грасгофа интенсивность течения возрастает, что приводит к искажению профиля концентрации, однако вертикальный градиент плотности в значительной мере сохраняется.

Исследование процессов перераспределения примеси между двумя емкостями показало, что профиль концентрации также сохраняется в значительной мере.
Процессы перетекания длятся в течении малого времени, порядка гидродинамического. Получены профили распределения концентрации в процессе конвективного течения.

Автор выражает благодарность проф. Б.Л. Смородину за полезные обсуждения и ценные комментарии. Исследования выполнены при финансовой поддержке Российского фонда фундаментальных исследований (№ 16-3160074).

\section{Список литературы}

[1] Евстратова К.И., Купина Н.А., Малахова Е.Е. Физическая и коллоидная химия. Высш. шк. 1990. С. 487.

[2] Фертман B.E. Магнитные жидкости. Высш. шк. 1988. C. 184.

[3] Donzelli D., Cerbino R., Vailati A. // Phys. Rev. Lett. 2009. Vol. 102. P. 10503.

[4] Глухов А.Ф., Путин Г.Ф. Установление равновестного барометрического распределения частиц в магнитной жидкости. Гидродинамика, 1999.

[5] Smorodin B., Myznikova B., Legros J. // Phys. Fluid. 2008. Vol. 20. P. AN 094102.

[6] Krauzina M.T., Bozhko A.A., Putin G.F. et al. // Phys. Rev. E. 2015. Vol. 91. P. 013010.

[7] Глухов А.Ф., Демин В.А., Мальгачева И.А. и др. // Известия Том. политех. ун-та. 2012. Т. 320. С. 41-45.

[8] Божко А.А. // Вестник Пермского ун-та. Сер. Физика. 2012. T. 3. № 21. C. 41-52.

[9] Shliomis M.I., Smorodin B.L. // Phys. Rev. E. 2005. Vol. 71.

[10] Smorodin B.L., Cherepanov I.N., Myznikova B.I. et al. // Phys. Rev. E. 2011. Vol. 84. P. 026305.

[11] Bernardin M., Comitani F., Vailati A. // Phys. Rev. E. 2012. Vol. 85. P. 066321.

[12] Shliomis M.I., Smorodin B.L. // J. Magn. Magn. Mater. 2002. Vol. 252. P. 197-202.

[13] Smorodin B.L., Cherepanov I. // J. Exper. Theor. Phys. 2015. Vol. 120. P. 319-326.

[14] Raikher Y., Shliomis M. // J. Magn. Magn. Mater. 1993. Vol. 122. P. 93-97.

[15] Гершуни Г.З., Жуховицкий Е.М., Непомнящий А.А. Устойчивость конвективных течений. М.: Наука, 1989. С. 320.

[16] Черепанов И.Н. // Вычислительная механика сплошных сред. 2016. Т. 9. № 2. С. 135-144. 
[17] Bozhko A.A., Putin G.F., Sidorov A.S. et al. // Magnetohydrodynamics. 2013. Vol. 49. P. 143-152.

[18] Нигматулин И.Р. Динамика многофазных сред. Ч. 1. М.: Наука, 1987. С. 464.

[19] Черепанов И.Н., Попов В.А. Экспериментальное исследование влияния концентрации на параметры наножидкости. Вестник Пермского ун-та. Физика. 2017. Т. 2. № 36. C. 26-32.

[20] Сидоров А.С. Конвективные автоколебания в вертикальном слое магнитной жидкости. Вестник Пермского ун-та. Сер. Физика. 2013. Т. 3. № 25. С. 50-53.

[21] Bozhko A., Putin G. // Microgravity Science and Technology. 2008. Vol. 21. P. 89-93.

[22] Ландау Л.Д., Лифиии, Е.М. Теоретическая физика. Гидродинамика. М.: Физматлит, 2001. Т. 6. С. 736.

[23] Рудяк В.Я. Современное состояние исследований вязкости наножидкостей. Вестник Новосибирского гос. ун-та. Cер. Физика. 2015. Т. 1. С. 5-22.

[24] Chen Y., Zhang L., Sun D. et al. // Microgravity Sci. Technol. 2016. Vol. 28. P. 95-100.

[25] Рудяк В.Я., Краснолуцкий С.Л. // ЖТФ. 2015. Т. 85 Вып. 6. C. 9-16. 